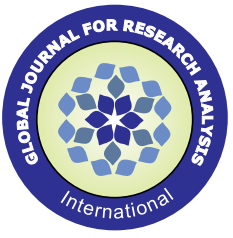

Dr. Nina M. Shah

Dr. Swati Mohanty*

\title{
A CASE REPORT OF SPLENIC ARTERY ANEURYSM
}

Associate Professor \& HOU, Department Of General Surgery, BJ Medical College, Civil Hospital Ahmedabad.

Third Year Resident, Department Of General Surgery, BJ Medical College, Civil Hospital Ahmedabad. * Corresponding Author

Dr. Hiral C. Chauhan Assistant Professor, Department Of General Surgery, BJ Medical College, Civil Hospital Ahmedabad.

\section{KEYWORDS :}

\section{INTRODUCTION}

True splenic artery aneurysms are the most common visceral artery aneurysms, yet are rare lesions seen in $0.1 \%$ to $0.8 \%$ of cases reviewed by autopsy or angiogram series. Concomitant visceral aneurysms are seen in 3\% of cases whereas non visceral aneurysms in $12 \%$.

\section{CASE REPORT}

A 42 year old female patient presented to civil hospital ahmedabad on $10^{\text {th }}$ september 2020 with chief complaint of abdominal distention since 15 years and repeated episodes of blood transfusion since the age of 15 years. Patient had a past history of road traffic accident at the age of 15 years in which she had lost right upper and lower limb. Since then she had history of repeated episodes of repeated hospitalisation for blood transfusions. Frequency of blood transfusion was once every 15 days. Patient was vitally stable. Icterus and Pallor present. Per abdomen: grade 3 spleenomegaly and abdominal distention present. Chest and abdomen x-ray normal.

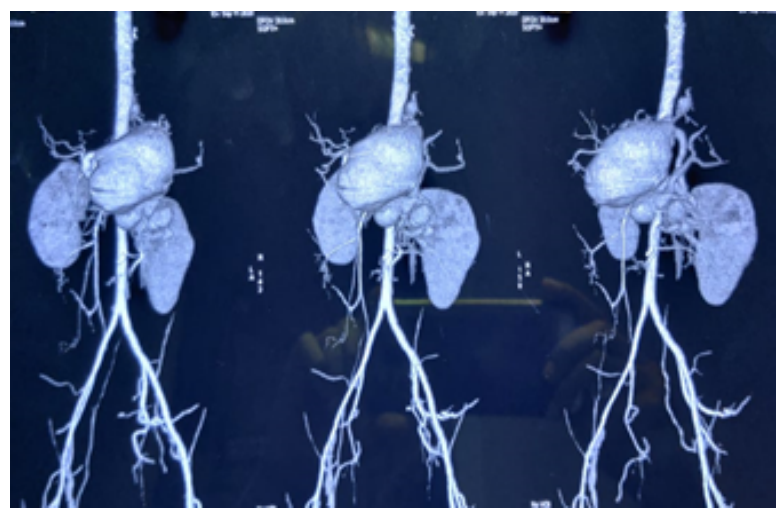

Figure l cect angiography of aorta

Usg abdomen pelvis: 59x105x71 mm sized cystic hypoechoic lesion with mixed internal vascularity in epigastric region possibility of aneurysm arising from splenic artery. Liver normal in size and altered echotexture s/o liver parenchymal disease. Portal vein $17 \mathrm{~mm}$ showing normal hepatopetal flow and respiratory phasic variation. Gall bladder well distended with few calculi largest $10-11 \mathrm{~mm}$. spleen enlarged $>20 \mathrm{~cm}$ showing multiple hyperechoic foci within p/o gamma gandy bodies. Multiple dilated and tortuous splenic hilar, peripancreatic, mesenteric and lienorenal collaterals.

Cect angiography abdomen pelvis: Large fusiform thin walled aneurysm of approximate size 57 × $97 \times 70 \mathrm{~mm}$ noted in the epigastric region originating from splenic artery just inferior to the stomach causing compression over stomach superiorly. Spleen appears enlarged in size measuring $26 \mathrm{~cm}$. Spleen is extending upto the midline in the umbilical region. Multiple gall bladder calculi (atleast 6-7) are noted, largest measuring $20 \mathrm{~mm}$. No evident of acute cholecystitis. Liver appear normal in size and enhancement. Aneurysm is posteriorly abutting to pancreas with severe volume reduction of body of pancreas. Head and uncinate process appears normal with changes of portal hypertension.

Decision was made to do aneurysm excision with spleenectomy and cholecystectomy.

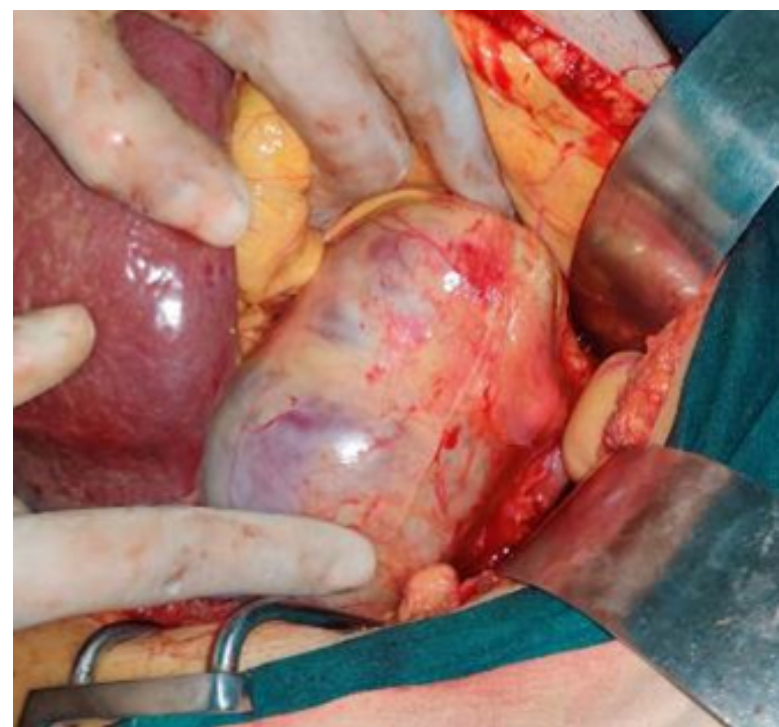

Figure2 splenic artery aneurysm

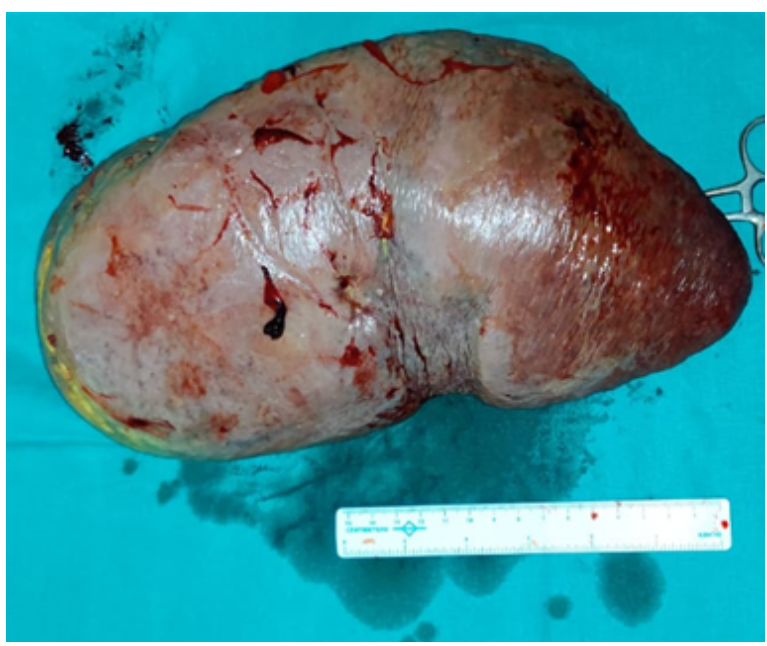

Figure3 specimen of spleen 
Intraoperatively: patient was explored from rooftop incision. After excision of aneurysm splenic artery was closed with prolene 5-0. Along with splenectomy, cholecystectomy was done uneventfully,Patient had been kept nbm for 1 day after which she was started on sips orally. The drain output in morrisson and splenic fossa in first week was average of 200 to $300 \mathrm{cc}$ serous pt was started on soft diet after 2 days. In second week drain output increased to 800 to $1000 \mathrm{ml}$ in both sides. She was started on tablet lasilactone $25 \mathrm{mg}$ 1-1-0 initially which was increased to 2-2-0 later on. Intermittent blockage trial of drain was done also antigravity drainage tried. On giving intermittent block trial main wound had serous discharge on which stoma bag was applied. Burst abdomen occured on pod 8 over left aspect of incision in which output was $100 \mathrm{cc}$ per day. But drain output was still average of $500 \mathrm{ml}$ on both sides. Burst abdomen was closed with secondary resuturing and Alternate suture removal was done in main wound on pod 10, on pod 15 morrison drain was removed and stitch was taken at the drain site. On pod 17 splenic fossa drain was removed and stitch taken over drain site. She had developed recurrent severe electrolyte imbalance episodes sodium $<120$ and potassium $<2.5$ for which correction was given.

\section{Histopathology s/o splenic artery aneurysm.}

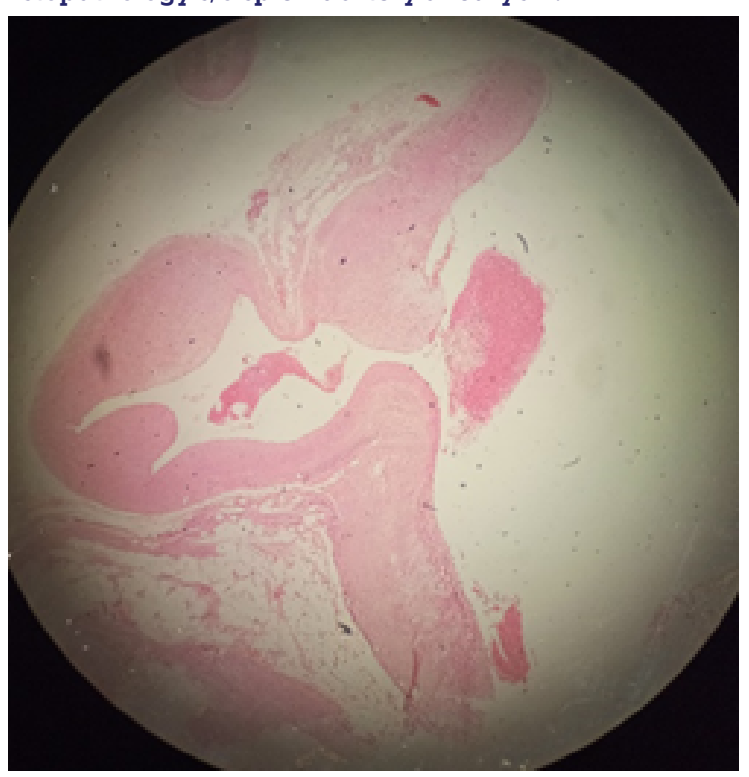

Figure 4 histopathology of aneurysm wall

Post operative usg showed partial portal vein thrombosis with changes of liver parenchymal disease. On pod 20, patient turned out to be covid positive and was shifted to covid ward for further management.

\section{DISCUSSION}

Splenic artery aneurysm more common in females than males 4:1 ratio. Etiology is hyperplasia of media and fragmentation of elastic lamina. Pregnancy and portal hypertension have increased risk of splenic artery aneurysm. Commonly seen in fibromuscular dysplasia, alpha 1 at deficiency, polyarteritis nodosa, marfan syndrome, ehler danlos syndrome, sle, scleroderma and hypertension.

Mainly found incidentally on cross sectional imaging and angiograms. $75 \%$ present in distal artery and $20 \%$ in middle of vessel. Rarely symptoms of epigastricor left upper quadrant pain, or vomiting can be seen. Occasionally patients present with abdominal pain followed 6 to 96 hrs later by hemodynamic instability. This "double rupture" phenomenon caused by initial bleeding with tamponade in lesser sac followed by subsequent delayed intraperitoneal haemorrhage.
Alternately aneurysm can erode into stomach, colon or pancreatic duct resulting in gi bleeding or into splenic vein resulting in arterioportal fistula.

False splenic artery aneurysm are less common than true aneurysms and associated with trauma, infection or inflammation. Most common etiology is pancreatitis with pseudocyst formation. Aneurysm more than $2 \mathrm{~cm}$ in size, symptomatic or growing on periodic imaging should be considered for treatment. Elective management: transcatheter endovascular techniques including embolization and stent graft placement are gold standard. Embolization done via coils, gelfoam, glue, thrombin, amplatzer vascular plug. If good collateral flow, efferent arterial flow occluded with balloon or embolized to enhance aneurysm thrombosis. Surgical options include proximal and distal artery ligation and aneurysm excision. Mortality associated with elective endovascular techniques and surgical management ranges from $0-2 \%$ and $3-5 \%$ respectively. In open surgery $20-30 \%$ perioperative mortality present.

\section{CONCLUSION}

Splenic artery aneurysm should be managed as soon as possible as an emergency as it always has a chance of rupture (6\%) which increases the mortality rates(36\%) due to its high pressure and high flow system. Spontaneous rupture of splenic artery aneurysms that are asymptomatic and are less than $2 \mathrm{~cm}$ in diameter is rare, especially in patients with no risk factors. A follow-up period of one year is recommended, but it may be extended if other medical risks are present. As far as possible endovascular techniques should be tried as a gold standard method but due to unavailability of facilities, should not be managed conservatively and requires vigorous management. Post surgery patient may develop complications like portal vein thrombosis but it can be managed later on. Rapidly increasing size, presence in a premenopausal woman, cirrhosis, and symptomatic aneurysm may warrant intervention, regardless of size.

\section{REFERENCES}

1. Abbas MA, Stone WM, Fowl RJ, Gloviczki P, Oldenburg WA, Pairolero PC Hallett JW, Bower TC, Panneton JM, Cherry KJ (2002)."Splenic arterya neurysms: two decades experience at Mayo clinic". Ann Vasc Surg. 16(4): 442449. PMID 12089631

2. Jamsheer NS, Malik M (2001). "Ruptured splenic artery aneurysm". Ann Saudi Med. 21(5-6):pp. 340-341. Accessed July 30, 2005.

3. Khosa, Faisal, MD. "Managing Incidental Findings on Abdominal and Pelvic CT and MRI, Part 2: White Paper of the ACR Incidental Findings Committee II on Vascular Findings." Journal of the American College of Radiology 10.10 (2013): 789-94.

4. Parrish J, Maxwell C, Beecroft J. Splenic Artery Aneurysm in Pregnancy. JOGC. 2015; 37(9):816-818. 